Chirurg 2016 $87: 345$

DOI 10.1007/s00104-016-0176-3

Online publiziert: 8. April 2016

(c) Springer-Verlag Berlin Heidelberg 2016

CrossMark

\section{Schrempf $\cdot$ M. Anthuber}

Klinik für Allgemein-, Viszeral- und Transplantationschirurgie, Klinikum Augsburg, Augsburg, Deutschland

\title{
Einfluss der neoadjuvanten RCT auf das Langzeitergebnis beim Ösophaguskarzinom und AEG
}

\section{Originalpublikation}

Shapiro J, van Lanschot JJ, Hulshof MC et al (2015) Neoadjuvant chemoradiotherapy plus surgery versus surgery alone for oesophageal or junctional cancer (CROSS): long-term results of a randomised controlled trial. Lancet Oncol 16: 1090-1098

\section{Hintergrund}

Das lokal fortgeschrittene Ösophaguskarzinom ist durch eine hohe Rate an lokalen und systemischen Rezidiven nach primärer Resektion gekennzeichnet. Die „ChemoRadiotherapy for Oesophageal cancer followed by Surgery Study“ (CROSS) vergleicht neoadjuvante Radiochemotherapie (RCT) und anschließende Resektion mit alleiniger Resektion bei Patienten mit Plattenepithelund Adenokarzinom des Ösophagus und des gastroösophagealen Übergangs (AEG, adenocarcinoma of the esophagus and junction). In der vorliegenden Arbeit werden die Langzeitergebnisse nach einer Nachbeobachtungszeit von mindestens 5 Jahren vorgestellt.

\section{Methoden}

Patienten mit resektablem, lokal fortgeschrittenem Karzinom des Ösophagus und des gastroösophagealen Übergangs (klinische Stadien T1N1M0 oder T23N0-1M0) wurden mittels Blockrandomisierung einer Behandlungsgruppe zugeteilt. Die Patienten erhielten entweder eine neoadjuvante RCT bestehend aus Carboplatin und Paclitaxel mit begleitender Bestrahlung (41,4 Gy) gefolgt von einer Resektion oder eine alleinige Resektion. Primärer Endpunkt war das Gesamtüberleben. Die Auswertung erfolgte mittels Intention-to-treat-Analyse.

\section{Ergebnisse}

Zwischen 2004 und 2012 wurden 386 Patienten aus 8 teilnehmenden niederländischen Zentren in die Studie eingeschlossen. 180 Patienten wurden einer neoadjuvanten RCT mit anschließender Resektion und 188 Patienten der alleinigen Resektion zugeteilt. Nach einer medianen Nachbeobachtungszeit von 84,1 Monaten zeigte sich ein signifikant längeres medianes Gesamtüberleben nach neoadjuvanter RCT und anschließender Resektion im Vergleich zur alleinigen Resektion (48,6 vs. 24,0 Monate; HR 0,68 [95\%-KI 0,53-0,88]). Sowohl für das Plattenepithel- als auch für das Adenokarzinom zeigte die univariate Analyse eine signifikante Verlängerung des medianen Gesamt- und des progressionsfreien Überlebens durch neoadjuvante RCT. In der multivariaten Analyse war für das Plattenepithelkarzinom weiterhin eine signifikante Reduktion der Gesamtmortalität durch neoadjuvante RCT nachweisbar (korrigierte HR 0,46 [95\%-KI 0,26-0,79]; p = 0,005), nicht jedoch für das Adenokarzinom (korrigierte HR 0,75 [95\%-KI 0,56-1,01]; p = 0,059).

\section{Diskussion und Fazit}

Die CROSS-Studie bestätigt eine Verlängerung des Gesamt- und des progressionsfreien Überlebens durch neoadjuvante RCT für Patienten mit Plattenepi- thel- und Adenokarzinom des Ösophagus. Bei relativ geringer Patientenzahl in der Plattenepithelkarzinomsubgruppe (23\%) war der Effekt auf die Gesamtmortalität stärker ausgeprägt. Das Signifikanzniveau wurde in der multivariaten Analyse in der Adenokarzinomsubgruppe knapp verfehlt. Unklarheit muss weiterhin über den Nutzen einer neoadjuvanten RCT in den frühen Stadien cT1 und cT2 bestehen, deren Anteil in der CROSS-Studie mit weniger als $20 \%$ gering ausfiel. Eine frühere Arbeit hatte unter Verwendung eines anderen RCT-Schemas eine erhöhte postoperative Mortalität und keinen Überlebensvorteil nach neoadjuvanter RCT für die Stadien I und II gezeigt [1]. Es wäre daher erforderlich, Kriterien zu etablieren, mit deren Hilfe Patienten identifiziert werden können, die tatsächlich von einer neoadjuvanten RCT profitieren.

\section{Korrespondenzadresse}

\section{Prof. Dr. M. Anthuber}

Klinik für Allgemein-, Viszeral- und Transplantationschirurgie, Klinikum Augsburg Stenglinstr. 2, 86156 Augsburg, Deutschland matthias.anthuber@klinikum-augsburg.de

Interessenkonflikt. M. Schrempf und M. Anthuber geben an, dass kein Interessenkonflikt besteht.

\section{Literatur}

\footnotetext{
1. Mariette C, Dahan L, Mornex F et al (2014) Surgery alone versus chemoradiotherapy followed by surgery for stage I and II esophageal cancer: final analysis of randomized controlled phase III trial FFCD 9901.J Clin Oncol 32:2416-2422
} 